\section{Pearls and pitfalls in genetic studies of migraine}

Cephalalgia

33(8) 6I4-625

(C) International Headache Society 2013

Reprints and permissions:

sagepub.co.uk/journalsPermissions.nav DOI: 10.1 I 77/0333 I024|3484988 cep.sagepub.com

(S) SAGE

\author{
Else Eising', Boukje de Vries', Michel D Ferrari', \\ Gisela $M$ Terwindt ${ }^{2}$ and Arn MJM van den Maagdenberg ${ }^{1,2}$
}

\begin{abstract}
Purpose of review: Migraine is a prevalent neurovascular brain disorder with a strong genetic component, and different methodological approaches have been implemented to identify the genes involved. This review focuses on pearls and pitfalls of these approaches and genetic findings in migraine.

Summary: Common forms of migraine (i.e. migraine with and without aura) are thought to have a polygenic make-up, whereas rare familial hemiplegic migraine (FHM) presents with a monogenic pattern of inheritance. Until a few years ago only studies in FHM yielded causal genes, which were identified by a classical linkage analysis approach. Functional analyses of FHM gene mutations in cellular and transgenic animal models suggest abnormal glutamatergic neurotransmission as a possible key disease mechanism. Recently, a number of genes were discovered for the common forms of migraine using a genome-wide association (GWA) approach, which sheds first light on the pathophysiological mechanisms involved.

Conclusions: Novel technological strategies such as next-generation sequencing, which can be implemented in future genetic migraine research, may aid the identification of novel FHM genes and promote the search for the missing heritability of common migraine.
\end{abstract}

\title{
Keywords
}

Migraine, gene, linkage, association, genome-wide, next-generation sequencing

Date received: 19 October 2012; revised: II March 2013; accepted: II March 2013

\section{Introduction: Migraine as a genetic disease}

Migraine is a common neurovascular brain disorder that is characterized by attacks of severe, unilateral, pulsatile headache that is often accompanied by nausea, vomiting, photo- and/or phonophobia (1). Women are three times more often affected than men (2). At least $12 \%$ of the general population suffers from recurrent migraine attacks (3). The presence or absence of an aura can precede the headache phase in one-third of patients, and distinguishes between migraine with aura (MA) and migraine without aura (MO) (1). The aura consists of transient mainly visual, sensory and speech-related symptoms, and is likely caused by cortical spreading depression (CSD) events, which are slowly propagating cortical waves of neuronal and glial depolarization $(4,5)$. The headaches are brought about by an activation of the trigeminovascular system that involves abnormal processing of signals from innervated blood vessels in the meninges to brainstem centers. Studies in experimental animals have indicated that CSD events may activate the relevant brainstem centers and thereby would link aura and pain mechanisms $(6-8)$, but such proof is lacking in humans.

Migraine has a strong genetic component. Population-based family studies showed that the familial risk of migraine is increased $(9,10)$. A contribution of genetic factors in migraine was also apparent from twin studies that showed a concordance twice as high in monozygotic versus dizygotic twins $(11,12)$. In fact, a

\footnotetext{
'Department of Human Genetics, Leiden University Medical Centre, The Netherlands

${ }^{2}$ Department of Neurology, Leiden University Medical Centre, The Netherlands

Corresponding author:

Arn MJM van den Maagdenberg, Departments of Human Genetics and Neurology, Leiden University Medical Centre, PO Box 9600, 2300 RC Leiden, The Netherlands.

Email: maagdenberg@lumc.nl
} 
large Dutch twin study revealed that genetic and environmental factors had an almost equally large contribution (13). This review highlights some of the recent advances and controversies in the search for migraine genes. Special attention is paid to the different methodological approaches used in genetic research in the (recent) past (linkage with markers, candidate gene association studies), the present (genome-wide association studies (GWAS)), and the future (next-generation sequencing (NGS)).

\section{Pearls in genetics}

\section{Gene identification by classical linkage analysis in monogenic familial hemiplegic migraine (FHM)}

A linkage approach, traditionally, has been most successful for the identification of causal genes in monogenic disorders. With linkage analysis the segregation of hundreds to several thousand genetic markers (earlier multi-allelic markers and more recently singlenucleotide polymorphisms (SNPs)) that are evenly distributed over the genome are compared with the co-occurrence of a disease trait in a family-based design. The fit is calculated as a logarithm (base 10) of odds (LOD) score that provides statistical proof that a trait locus resides at a given chromosomal location. Subsequent sequencing of candidate genes in the linked chromosomal region is needed to identify the causal gene mutation in patients that should not be present in control subjects. In migraine genetics the linkage approach was successfully applied for FHM, a rare autosomal dominant subtype of MA that is characterized by a transient hemiparesis during the aura phase of the attack, which led to the identification of three genes.

FHM genes CACNA1A (FHM1) (14)), ATP1A2 (FHM2) (15) and SCN1A (FHM3) (16) all encode proteins that affect ion transport in the brain. Their gene products are subunits of certain voltage-gated calcium channels, sodium-potassium ATPases, or voltage-gated sodium channels, respectively, and analysis of gene mutations identified a major role for glutamatergic neurotransmission in migraine (Figure 1) (17). The identification of FHM genes has had an enormous impact on diagnosing patients as the presence of a gene mutation, with its large effect size, confirms the clinical diagnosis. Genotype-phenotype correlation studies revealed that mutation carriers can suffer from a wide variety of associated symptoms, including cerebellar ataxia, seizures, mental retardation, mild head trauma-induced edema that can be fatal (e.g. in case of the FHM1 S218L missense mutation (18)), or even "elicited repetitive daily blindness" that can occur apart from FHM attacks in some carriers of FHM3 mutations Q1489H and F1499L (19,20). Notably, certain mutations in FHM genes do not cause FHM, but seemingly unrelated brain disorders, such as episodic ataxia type 2 or spinocerebellar ataxia type 6 (in the case of $C A C N A 1 A)(14,21)$ or severe childhood epilepsies, such as severe myoclonic epilepsy of infancy and generalized epilepsy with febrile seizures plus (in the case of $S C N 1 A$ ) (22), which provides additional opportunities to investigate how FHM gene mutations affect brain function in a disease-specific manner.

If hemiplegic migraine occurs not in a family but in an isolated case, it is called sporadic hemiplegic migraine (SHM). Apart from the absence of an affected close relative, the diagnostic criteria and attacks are identical to those in FHM (23). Most screens of FHM genes in SHM patients revealed mutations, predominantly in ATP1A2, in only a small proportion of patients (24-27). Riant and colleagues (28) studied a group of 25 SHM patients with an age of onset before 16 years, of whom 18 patients had additional symptoms such as epilepsy, learning difficulties, cerebellar ataxia, and/or coma. Perhaps surprisingly, in no fewer than 23 patients, mutations in CACNA1A and $A T P 1 A 2$ were identified. Three-quarters of the mutations had occurred de novo and mutation carriers thus represent the first patients of new FHM families. A recently identified de novo G715R ATP1A2 mutation was found in a 6-year-old SHM patient with prolonged hemiparesis, which resolved after four weeks and required rehabilitation (29); this further supports the observation that particularly de novo mutations are present in severely affected patients. The question remains what is causing SHM in the patients who do not carry an FHM gene mutation, which is a rather large proportion of patients in most studies. Possibilities are that either other FHM genes may cause hemiplegic migraine in those patients or that SHM (especially when the phenotype is not severe) is due to a combination of multiple low-risk genetic variants, similar to what is predicted to occur in common migraine. Support for the latter hypothesis comes from the observation that MA is frequent in families of SHM patients (30). Also, it would fit a view of migraine being a spectrum of disorders. It certainly stresses the importance of taking a reliable family history in patients with suspected hemiplegic migraine and of follow-up of these patients. This is also exemplified by a recent clinical follow-up study of 19 Dutch SHM patients, which showed that in a proportion of SHM patients the diagnosis changed over time to FHM (31).

Genetic studies that tried to find evidence for involvement of FHM genes in common forms of migraine are essentially negative, including the most systematic and largest study that screened several thousand DNA polymorphisms in more than 150 ion 


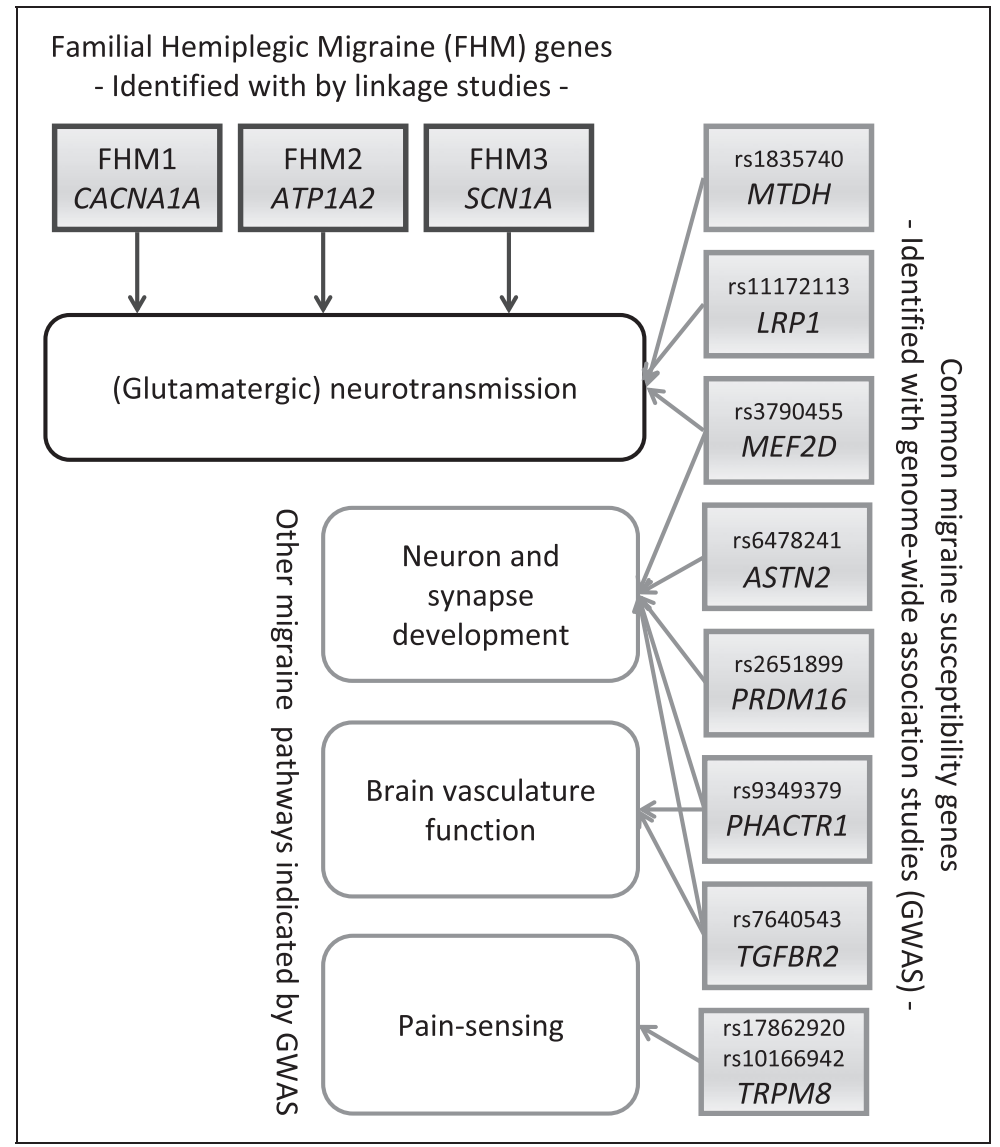

Figure I. Genes and pathways involved in familial hemiplegic migraine (FHM) and common migraine. FHM genes CACNAIA (FHMI), ATPIA2 (FHM2) and SCNIA (FHM3) are involved in the regulation of (glutamatergic) neurotransmission. Common migraine genes MTDH, LRPI and MEF2D that are indicated by genome-wide association studies (GWAS) hits seem to be involved in this pathway as well. Additional common migraine genes from the GWAS indicate neuron and synapse development (PHACTRI, ASTN2, PRDMI6, TGFBR2), brain vasculature function (TGFBR2, PHACTRI) and pain-sensing (TRPM8) as additional migraine-relevant pathways.

transporter genes in close to a thousand Finnish MA patients (and several thousand additional migraine patients in the replication phase of initial slightly positive association finding) (32). Of note, a similar-sized study in epilepsy also did not find genetic support for the involvement of ion transporter genes (33). This may seem paradoxical, also in light of the efficacy of antiepileptic drugs in epileptic and migraine patients, which act on neurotransmitter and ion channel pathways. A possible explanation might be that disease risk in these disorders is conferred by other - perhaps regulatory genes that control neurotransmitter and ion pathways in a more subtle manner than the ion transporters themselves.

\section{Functional characterization of FHM mutations in cellular and transgenic mouse models}

The large effect sizes of gene mutations in monogenic FHM make an investigation of their functional consequences in cellular and transgenic animal models feasible. Most cellular studies showed that FHM1 mutations exert gain-of-function effects by shifting neuronal $\mathrm{Ca}_{\mathrm{V}} 2.1$ channels' voltagedependence toward more negative membrane potentials while enhancing channel open probability (34), although loss-of-function effects have been reported as well $(35,36)$. Loss of glial $\mathrm{Na}^{+} / \mathrm{K}^{+}$ATPase function seems the most likely mechanism for FHM2 mutations (37). Most FHM3 mutations seem to exert loss-of-function effects of $\mathrm{Na}_{V} 1.1$ sodium channels that seem to primarily affect inhibitory neurons, but gain-of-function effects have been proposed for FHM3 mutation L263V that is associated with a combined seizure FHM phenotype in the majority of mutation carriers (38-40). Taken together, these cellular studies of FHM mutations predict increased neurotransmitter and potassium ion levels at the synaptic cleft, especially after high-intensity neuronal firing, which would facilitate cortical 
spreading depression (CSD) (41), and thereby could explain the migraine aura.

In vivo consequences of FHM mutations at the organism level can be investigated with the use of socalled knock-in (KI) mouse models by introducing human pathogenic mutations in the endogenous gene using gene-targeting technology. For FHM1, two KI models have been generated that harbor gainof-function missense mutations R192Q or S218L $(42,43)$. In line with the clinical phenotype in mutation carriers, only S218L mice exhibit the complex phenotype of cerebellar ataxia, susceptibility to seizures and delayed cerebral edema after minor head trauma (43). Mice with the milder R192Q mutation show no overt phenotype (42), although signs of abnormal facial expression that may reflect a pain phenotype, which was prevented by administrating a serotonergic migraine drug, have been reported (44). At the neurobiological level, mice of both mutant strains revealed increased neuronal calcium influx and (cortical) neurotransmitter release, as well as an increased susceptibility to $\operatorname{CSD}(42,43,45,46)$. These features were all more prominent in the severer S218L mutant. In line with the female preponderance in migraineurs, female mutant mice are more susceptible to CSD than male mice (45). Recently, increased susceptibility to CSD was also reported in heterozygous KI mice that harbor the loss-of-function FHM2 mutation W887R (47). The experiments cannot be conducted in (adult) homozygous FHM2 mutant mice as the mice die directly after birth, identical to the fate of homozygous Atpla2 knockout mice (48).

\section{GWAS revealed first susceptibility genes in common migraine}

During the last few years, GWAS have become the most used approach to identify genes that confer susceptibility to complex disorders. In a GWAS, hundreds of thousands of SNPs that are distributed over the genome are tested in a hypothesis-free manner for association with a disease trait. For each SNP, the allele frequencies are compared between cases and controls. Significant differences in allele frequency either pinpoint the SNP itself as a genetic susceptibility factor or provide statistical evidence that a causal gene variant is in close vicinity. The latter implies that the tested SNP and the causal variant do not segregate independently; in genetic terms they are in linkage disequilibrium. To sufficiently correct for multiple testing (i.e. for the hundreds of thousands of tests), only SNPtrait associations with $p$ values below $5.0 \times 10^{-8}$ are considered genome-wide significant. As a direct consequence, GWA studies require testing of several or many thousand cases and controls to have sufficient power. Importantly, initial positive association findings need to be confirmed in independent case and control cohorts before meaningful conclusions about the associations can be drawn.

At this moment, three major migraine GWA studies have been reported and a summary of the main results is presented in the Table 1 and in Figure 1. The first GWA study investigated 2748 European clinic-based MA cases and more than 10,000 matched controls $\left(49^{* *}\right)$. Only one SNP, rs 1835740, which is located on chromosome $8 \mathrm{q} 22.1$, showed robust significant association with the trait $\left(p=5.38 \times 10^{-9} ;\right.$ odds ratio $=1.23 ; 95 \%$ confidence interval (CI) 1.15-1.32). The SNP is located between the $M T D H$ (metadherin or astrocyte-elevated gene (AEGI)) and the $P G C P$ (plasma glutamate carboxypeptidase) genes. Expression quantitative trait analysis that correlates gene expression to genotypic variation in specific cell lines revealed that the associated allele of SNP rs1835740 correlates with increased gene expression level of $M T D H$. Functional MTDH analyses from cancer research suggest that MTDH acts through activation of NF- $\kappa \mathrm{B}$ and has an inhibitory effect on the expression of cerebral glial glutamate transporter EAAT2 (50-53). Reduced glial EAAT2 levels would predict increased glutamate levels in the synaptic cleft, which would hint toward a role for glutamate neurotransmission in common migraine, similar to the disease mechanism that surfaced from FHM research. Notably, recent pharmacological approaches to target glutamate receptors for migraine treatment seem in line with these genetic findings (54).

The second major GWAS was conducted as part of the Women's Genome Health Study and involved 5122 migraine cases and more than 18,000 controls from the general population $\left(55^{* *}\right)$. After including the replication data, three loci became genome-wide significant, suggesting TRPM8, LRPI and PRDM16 as migraine susceptibility genes. The association findings were strongest for TRPM8. TRPM8 is a calcium ion channel protein that is located on peripheral afferents of sensory neurons and a subset of trigeminal ganglion neurons and is a sensor for cold and cold-induced burning pain $(56,57)$. TRPM8 is a target in animal models of neuropathic pain (58). LRPI encodes a lipoprotein receptor that seems to modulate neuronal glutamate signaling by astrocytic cycling of tissue-type plasminogen activator (59). A possible role for PRDM16 in migraine pathophysiology is less clear as the gene is implicated in brown fat development (60) although recent studies indicated a possible role in neuronal development as well $(61,62)$. The third GWA study was conducted in clinic-based MO patients (63**). The study included 2326 patients and almost 5000 controls, and identified four novel migraine susceptibility 
Table I. Migraine loci and genes identified by genome-wide association studies.

\begin{tabular}{|c|c|c|c|c|}
\hline $\begin{array}{l}\text { Chromosomal } \\
\text { locus }\end{array}$ & $\begin{array}{l}\text { Associated DNA } \\
\text { variant (and } \\
\text { implicated gene) }\end{array}$ & $\begin{array}{l}\text { Cohort }^{\mathrm{a}} \text { and } \\
\text { migraine type }\end{array}$ & $\begin{array}{l}p \text { value }^{\mathrm{b}} \text { (odds ratio } \\
(95 \% \text { confidence interval)) }\end{array}$ & Reference $^{b}$ \\
\hline $8 q 22.1$ & rsI835740 (MTDH) & Clinic-based MA & $1.69 \times 10^{-11}(1.18(1.13-1.24))$ & $(49 * *)$ \\
\hline \multirow[t]{4}{*}{$2 q 37$} & rsI7862920 (TRPM8) & Clinic-based MA & $1.26 \times 10^{-5}(0.78(0.69-0.87))$ & $(49 * *)$ \\
\hline & rsI0I66942 (TRPM8) & Clinic-based MO & $5.97 \times 10^{-9}(0.77(0.70-0.84))$ & $\left(63^{* *}\right)$ \\
\hline & & $\begin{array}{l}\text { Population-based } \\
\text { migraine }\end{array}$ & $5.50 \times 10^{-12}(0.85(0.82-0.89))$ & $\left(55^{* *}\right)$ \\
\hline & & Clinic-based MO & $9.83 \times 10^{-13}(0.87(0.73-0.84))$ & $\left(63^{* *}\right)$ \\
\hline \multirow[t]{2}{*}{$2 q|3-q| 4$} & rsIII72II3 (LRPI) & $\begin{array}{l}\text { Population-based } \\
\text { migraine }\end{array}$ & $\begin{array}{l}4.30 \times 10^{-9} \\
\quad(0.90(0.78-0.93))\end{array}$ & $\left(55^{* *}\right)$ \\
\hline & & Clinic-based MO & $\begin{array}{l}2.97 \times 10^{-8} \\
\quad(0.86(0.81-0.91))\end{array}$ & $\left(63^{* *}\right)$ \\
\hline Ip36.23-p33 & rs265I899 (PRDM I6) & $\begin{array}{l}\text { Population-based } \\
\text { migraine }\end{array}$ & $\begin{array}{l}3.80 \times 10^{-9} \\
\quad(1.11(1.07-1.15))\end{array}$ & $\left(55^{* *}\right)$ \\
\hline$|q| 2-q 23$ & rs3790455 (MEF2D) & Clinic-based MO & $7.06 \times 10^{-11}(1.20(1.14-1.27))$ & $\left(63^{* *}\right)$ \\
\hline 6p24.I & rs9349379 (PHACTRI) & Clinic-based MO & $3.20 \times 10^{-8}(0.86(0.8 I-0.9 I))$ & $\left(63^{* *}\right)$ \\
\hline $3 p 22$ & rs7640543 (TGFBR2) & Clinic-based MO & $1.17 \times 10^{-9}(1.19(1.13-1.26))$ & $\left(63^{* *}\right)$ \\
\hline $9 q 33.1$ & rs647824I (ASTN2) & Clinic-based MO & $3.86 \times 10^{-8}(1.16(1.11-1.23))$ & $\left(63^{* *}\right)$ \\
\hline
\end{tabular}

${ }^{a}$ Cohort type can be clinic based (i.e. cases were collected through specialized headache clinics) or population based (i.e. cases were collected from a cohort).

${ }^{\mathrm{b}} P$ value of the meta-analysis of the initial and replication cohorts combined; except for rs 17862920 in the clinic-based MA study, the $p$ value represents the discovery cohort only. MA: migraine with aura; MO: migraine without aura: migraine: unspecified migraine type.

genes: MEF2D, TGFBR2, PHACTR1 and ASTN2, of which the latter two had less convincing $p$ values. In addition, this study confirmed a role for TRPM 8 and LRPI in migraine pathophysiology. At this moment, it is speculative how the novel genes may affect migraine pathophysiology. Of note, $M E F 2 D$ encodes a transcription factor that controls synapse development (64). Furthermore, one of the target genes of MEF2D encodes pituitary adenylate cyclaseactivating polypeptide-38 (PACAP-38), which may modulate excitatory synaptic transmission and can trigger migraine-like attacks in persons with MO (65). TGFBR2 encodes a serine-threonine kinase that is involved in cell proliferation and differentiation, as well as extracellular matrix production. A vascular role of TGFBR2 in migraine can be hypothesized from the observation that missense mutation p.Arg460His in TGFBR2 causes familial aortic dissection as well as migrainous headaches in 11 of 14 mutation carriers in a large multigenerational family (66). Although still highly speculative, PHACTR1, which is a member the PHACTR/scapinin family, might play a role in synaptic activity and synapse morphology or in endothelial cell functioning (67).

As the odds ratios observed with GWA studies in complex diseases generally are very small, ranging from 1.05 to 1.25 , the identified genetic variants have no direct clinical relevance for patient care. The same is true for migraine. This is not surprising since variants identified by GWA studies are present in a considerable proportion of healthy subjects. The variants occurred early in human evolution and it is therefore unlikely that they would have survived during evolution if they would have affected disease susceptibility in a dramatic manner. As many disease variants are located outside genes, for many of them it is not even immediately evident which biological pathways may be affected in patients. Still, hundreds to thousands of genes that have surfaced from the hundreds of GWA studies for a plethora of complex disorders provided first important insight in possible disease mechanisms. The low effect size is a complicating factor and considerably hampers straightforward functional analysis of diseaseassociated gene variants. Multiple ways have been proposed to bypass this downside of GWAS findings. For instance, one could perform biological pathway analyses of groups of related genes in specific pathways, guided by GWAS findings (68). Alternatively, one could investigate the function of the identified genes themselves for their consequence on disease-relevant pathways by modulating their expression (i.e. overexpression or knockdown) in various cellular and/or animal models (e.g. zebrafish or mouse) (69). Surely, development of more sophisticated models, which may include differentiated 
human-induced pluripotent stem cells as has been proposed for schizophrenia (70), seems needed for a meaningful functional evaluation of GWAS gene hits.

\section{Pitfalls}

\section{Classical linkage approach in common forms of migraine}

Whereas classical linkage analysis in monogenic FHM has led to the identification of several migraine genes, similar studies investigating common forms of migraine were not so successful. Although many chromosomal regions were identified that seem to harbor migraine susceptibility genes, no migraine genes were discovered (71). The most likely reason is that the genetic make-up in migraineurs with common migraine types is more complex with many genetic factors determining disease susceptibility together with environmental factors. Migraine susceptibility loci that reside on chromosomes 4q21-q24 (72,73) and 10q22-q23 $\left(74,75^{* *}, 76\right)$ seem most promising, also because these loci were found in more than one study. Still, the area of classical linkage approach led to potentially important novel ideas for phenotyping patients for genetic studies by challenging the classically used end-diagnoses of MA and MO as defined by the International Criteria of Headache Disorders (ICHD-II) (1). Using "latent class analysis" (LCA) that involves complex statistical empirical clustering based on factor analysis combining information on several migraine symptoms $\left(77^{* *}\right)$ or analyzing individual migraine sub-traits in "trait component analysis (TCA)" $\left(75^{* *}\right)$ may yield better results in future genetic migraine studies. Genetic studies of other complex disorders (e.g. schizophrenia and attention-deficit/hyperactivity disorder (ADHD)) have already indicated that individual sub-traits may have a higher heritability than the end-diagnosis itself $(78,79)$.

\section{Candidate-gene association studies in migraine}

Before the GWAS era, a frequently used alternative to family-based classical linkage studies has been the investigation of one (or sometimes a few) DNA variants in candidate genes that had emerged from preexisting knowledge. Many genes of the dopaminergic and serotonergic systems, hormone receptors and genes in inflammatory pathways were investigated (for a review, see Proudfoot et al. (57)). Although candidate gene-based association studies, in theory, present a powerful tool, they did not yield robust genetic associations. Most studies have one or more methodological issues with respect to small sample size, selection of cases and controls, insufficient correction for multiple testing, and/or incapability to replicate findings in independent populations. The best replicated genetic association is the C677T polymorphism in the 5,10-methylenetetrahydrofolate reductase (MTHFR) gene as can be concluded also from two meta-analyses that showed an association of the Tallele with MA, but not MO $(80,81)$. However, neither several large well-designed studies $(82,83)$ nor GWAS data found support for a genetic association. Regardless of lack of genetic evidence, a role for $M T H F R$, which codes for an enzyme of the homocysteine and folate metabolism (84), is appealing as high homocysteine levels (like seen in carriers of the T-allele) may induce vascular endothelial dysfunction and thereby increase migraine risk (85).

\section{Caution is needed when sequencing candidate genes}

Where hypothesis-driven candidate gene association studies in common disorders have led to genetic associations that could not be replicated - and most now are considered false-positive findings - the same may apply to candidate gene-sequencing studies in monogenic disorders if there is no sufficient evidence from replication efforts and if one is not cautious enough about the interpretation of results.

For example, common diseases (or symptoms of these diseases) such as migraine may co-occur just by chance with rare disorders. This fact may have confounded some of the disease implications of a homozygous S982NfsX4-truncating SLC4A4 mutation that resulted in a non-functional sodium bicarbonate cotransporter NBCel protein and was identified in two sisters with proximal renal tubular acidosis (pRTA) and ocular abnormalities (86). Whereas mutations in SLC4A4 are a well-known cause of proximal renal tubular acidosis (87), because both sisters also had hemiplegia and migraine attacks, the SLC4A4 gene was also presented as a possible recessive hemiplegic migraine gene. Two of six family members who were heterozygous for the SLC4A4 mutation had migraine (i.e. one had MA, the other had MO), which is not unexpected given the very high frequency of migraine in the general population. Although interesting as an isolated case, the finding may just be a spurious association in spite of the interesting hypothesis that NBCe1 dysfunction may disturb synaptic $\mathrm{pH}$ in astrocytes and thereby might affect migraine pathophysiology. The fact that four other homozygous SLC4A4 mutations, in additional to pRTA, cause different migraine phenotypes (i.e. hemiplegic migraine with episodic ataxia, MA, or MO (twice)), seems to add doubt that homozygous SLC4A4 mutations specifically cause hemiplegic migraine, and cannot be considered sufficient replication. It is more likely that (hemiplegic) 
migraine in these patients is caused by a yet uncovered gene defect.

Although direct sequencing of candidate genes, theoretically, is an appealing genetic approach to identify causal disease genes, one would predict that this is successful only in rare monogenic disorders, which are caused by single gene mutations with a high effect size. Recent large-scale sequencing studies (i.e. NGS of all coding exons in the genome) indicated that each of us, on average, carries a handful of novel nonsense, non-functional variants, which implies that the majority of these variants may be well tolerated (90). According to their publication, Lafrenière and colleagues selected the KCNK18 gene, which encodes the TRESK protein, based on the fact that this member of the two-pore domain family of potassium channels is involved in control of neuronal excitability, as a candidate gene for a multigenerational MA family (88). The F139WfsX24-truncating mutation that was identified results in a functionally inactive TRESK potassium channel protein and seems to fully explain migraine in this single MA family. No further causal evidence came from testing more than 600 additional migraine families. Is this sufficient proof for $K C N K 18$ as a migraine gene? Perhaps not, as the same authors showed that the C110R TRESK missense mutation, which also results in a non-functional copy of the KCNK18 gene, was identified in migraineurs as well as control individuals (89). A logical conclusion seems that non-functional TRESK copies are tolerated and do not lead to disease. KCNK18 mutations may still turn out genetic modifiers of a migraine phenotype, but it is not logical that it explains the clear-cut autosomal dominant inheritance in their original publication. The identification of the TRESK F139WfsX24 mutation seems to originate from a larger study in which 110 unrelated migraine patients were sequenced for 150 ion transporter genes (91), which may have considerably increased the chance of finding a nonfunctional mutation such as F139WfsX24 that does not have to be disease causing. The more likely scenario is that MA in the presented family is caused by a different gene mutation, perhaps in relative close proximity on chromosome 10q25. Regardless of the genetic doubt about causality, TRESK remains an interesting possible migraine target already because of its role in neuronal excitability (92).

\section{Open questions for the future}

\section{Gene identification with NGS}

The identification of three FHM genes did not end the gene hunt for this monogenic migraine type as there are many hemiplegic migraine patients in whom no causal gene mutation has been identified. Although hemiplegic migraine is a clinical diagnosis (sometimes compromised when confused with epilepsy or basilar migraine), knowledge of the disease-causing mutation can reassure clinicians and patients of that diagnosis. Moreover, it provides the direct possibility for genetic testing in additional family members. Finally, given the clinical variation observed with certain hemiplegic gene mutations, genetic testing provides information on possible severe clinical outcomes, such as a mild head-trauma-induced lethality (in the case of $\mathrm{S} 218 \mathrm{~L}$ in the $\mathrm{Ca}_{\mathrm{V}} 2.1$ channel gene $C A C N A 1 A(93))$ that in many cases would otherwise remain unknown. Moreover, knowledge of the neurobiological mechanisms of known and tobe-identified FHM genes will add further insight to the pathology of FHM, and likely also other migraine types. Current genetic knowledge seems to indicate that monogenic FHM and complex common migraine are caused by different gene variants/mutations with different effect sizes (Figure 2) and may share (at least in part) similar disease mechanisms, such as glutamatergic neurotransmission.

Gene identification capabilities have advanced dramatically in the last couple of years, as truly large-scale sequencing has become feasible by technical advances known as NGS. Whereas until recently genes were individually scrutinized for mutations with low-throughput Sanger dideoxy sequencing, it is now possible to costeffectively sequence all coding regions of proteins (i.e. exome sequencing) in a single experiment. In the very near future it will become (financially) feasible to sequence the entire genome (i.e. whole-genome sequencing) of multiple patients for research purposes $(94 * *)$. These novel possibilities will affect foremost monogenic disorders, as the inheritance pattern in these disorders will assist in selecting the most likely causal gene mutation (95**). Importantly, NGS will allow gene identification in FHM families that were too small for classical linkage analysis. It will be challenging though to uncover causal mutations from the vast amount of DNA variants that exome or genome sequencing produces. Sophisticated procedures for data pooling, bioinformatic filtering and variant prioritization methods will be essential to optimally harvest from this novel technology. Until now, most progress has been made with recessive disorders or sporadic disorders in which the mutation presents as de novo (i.e. is present in the patient but absent in unaffected parents). A recent successful example is sporadic alternating hemiplegia of childhood (AHC), which is a rare, severe neurodevelopmental syndrome characterized by recurrent hemiplegic episodes and distinct neurologic manifestations (96). Some $70 \%$ of AHC patients were shown to have a heterozygous de novo missense mutation in the ATPIA3 gene $\left(97 * *, 98^{* *}\right)$ that belongs to the same family as the 


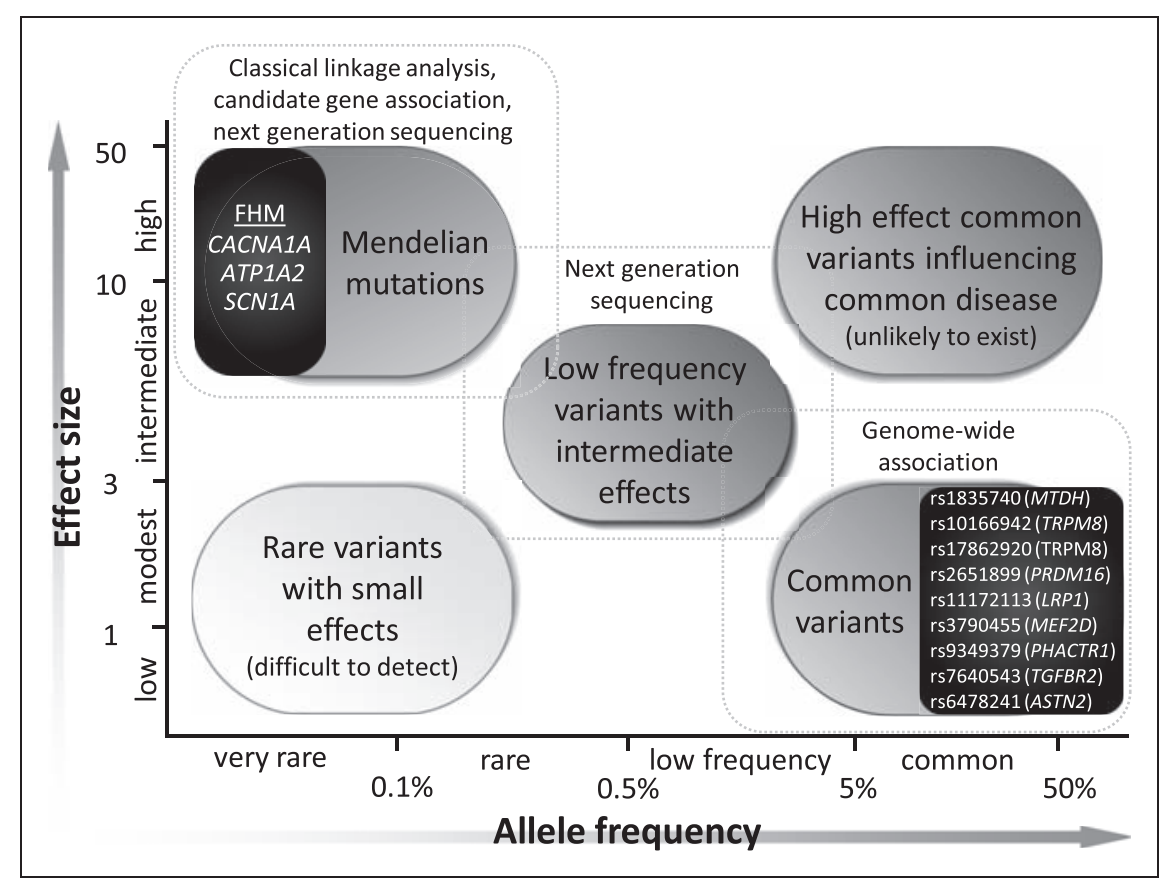

Figure 2. Different categories of genetic variation exist based on the frequency and effect size of a genetic variant. For rare variants with high effect sizes, until recently the classical linkage approach (combined with Sanger sequencing to identify the causal mutation) has been the method of choice for disease gene identification. This led to the identification of CACNAIA, ATPIA2 and SCNIA genes in familial hemiplegic migraine (FHM). Nowadays, next-generation sequencing (i.e. exome and whole-genome sequencing) has replaced the classical linkage approach. Only a few examples (e.g. complement factor $\mathrm{H}$ in age-related macular degeneration (I02)) are known of genetic factors with a high effect size and allele frequency. Common variants with a low effect size are typically identified with genome-wide association (GWA) studies. Examples of associated single-nucleotide polymorphisms (SNPs) in migraine are shown. Rare variants with a low effect size are currently out of reach; extensive whole-genome sequencing is required for identification of these variants. Discovery of low-frequency variants with medium-size effects might be identified in the next few years using nextgeneration (re-)sequencing. Adapted from Manolio et al. (99**).

FHM2 ATP1A2 gene. The real challenge for the future will be to successfully apply NGS to complex disorders and identify causal gene variants with a moderate effect size (i.e. larger than those of GWA studies, but smaller than those in monogenic diseases). For these genetically complex, polygenic disorders, it is far from clear whether analyzing the segregation of the large number of possible causal variants in family-based designs will be of sufficient help to pinpoint causal disease variants.

\section{Recommendation for future studies}

The methodology of GWA has been adopted widely in the genetic arena and has led to the identification of thousands of loci (and genes) for a wide variety of common diseases, including migraine. Hypothesis-free GWA studies essentially made candidate gene association studies redundant and definitely raised the bar for them as no genetic association study can do without an independent confirmation in other samples in the same study. Despite the obvious strength of GWA in identifying disease genes, the methodology, in some respects, did not live up to its expectation. Mainly because predicting individual genetic risk of disease from GWAS results is limited. In theory, one should be able to identify individuals with multiple-risk alleles who are at higher risk, but these calculations are not meaningful when only a fraction of the disease genes is known. If one calculates the sum of the effects of all loci discovered for a trait to obtain the combined genetic influence, at best one explains only $\sim 20 \%$ of the heritability of the trait. This implies that $\sim 80 \%$ of the heritability is still missing and not detected by the GWAS approaches used to date $\left(99^{* *}\right)$. This raises the serious question of where the missing heritability is hidden, and how this can be found. There are several possibilities. It may be that many of the common causal alleles simply have very small effect sizes that can only be detected in a GWAS with much larger sample sizes of cases and controls (i.e. with tens of thousands of individuals each). Also, causal variants may be rare and therefore not covered on commercial SNP chips used for GWA studies. These rare alleles are thought to have a larger effect size and can hopefully be detected by future NGS studies. Defining intermediate and/or functional phenotypes, such as an altered response to a migraine 
trigger-like nitric oxide donor (100), may be particularly useful for evaluating the genetic contribution of these rarer variants with potentially greater functional effects. Some of the heritability may also be explained by rare copy number variations as seems the case for neuropsychiatric disorders, such as schizophrenia $\left(101^{* *}\right)$. Finally, one has to take into account mechanisms such as epistasis, gene-environment interactions, and epigenetics. Epistasis can best be explained as variant-variant interactions in which one allele positively or negatively influences another allele, meaning that certain single SNPs with no or low marginal effects may have larger effects only in combination with another SNP (or SNPs). The potential of epistasis has not yet been investigated in migraine, also because the analysis of epistatic interactions suffers from a substantially increased multiple testing burden. Analyses of gene-environment interactions also have been understudied in GWAS, primarily because of lack of data on environmental exposures, which would require large prospective cohorts. Finally, epigenetic modifications, i.e. modifications to the genome other than changes in the DNA sequence, such as DNA methylation and histone modifications, may affect disease susceptibility by influencing gene expression, e.g. after exposure to environmental triggers. An important lesson for the future seems that one would need all of the above-mentioned approaches to further unravel the genetic background of common diseases, such as migraine. Notably, advancing the understanding of the genetic background of diseases that are co-morbid with migraine, e.g. epilepsy and depression, may become instrumental, not only for the understanding of the co-morbid relation, but perhaps also of migraine pathophysiology itself. Undoubtedly, the coming years will be very exciting as the DNA technologies that have been developed in the last few years, e.g. GWAS, exome sequencing and whole-genome sequencing, will be exploited to the full to uncover migraine genes and mechanisms.

\section{Funding}

This research received no specific grant from any funding agency in the public, commercial, or not-for-profit sectors.

\section{Conflict of interest}

None declared.

\section{References}

1. Headache Classification Subcommittee of the International Headache Society. The International Classification of Headache Disorders. 2nd ed. Cephalalgia 2004; 24: $1-160$.
2. Lipton RB, Stewart WF, Diamond S, et al. Prevalence and burden of migraine in the United States: Data from the American Migraine Study II. Headache 2001; 41: 646-657.

3. Launer LJ, Terwindt GM and Ferrari MD. The prevalence and characteristics of migraine in a populationbased cohort: The GEM Study. Neurology 1999; 53: 537-542.

4. Lauritzen M. Pathophysiology of the migraine aura. The spreading depression theory. Brain 1994; 117: 199-210.

5. Lauritzen M, Dreier JP, Fabricius M, et al. Clinical relevance of cortical spreading depression in neurological disorders: Migraine, malignant stroke, subarachnoid and intracranial hemorrhage, and traumatic brain injury. J Cereb Blood Flow Metab 2011; 31: 17-35.

6. Bolay H, Reuter U, Dunn AK, et al. Intrinsic brain activity triggers trigeminal meningeal afferents in a migraine model. Nat Med 2002; 8: 136-142.

7. Zhang X, Levy D, Noseda R, et al. Activation of meningeal nociceptors by cortical spreading depression: Implications for migraine with aura. J Neurosci 2010; 30: 8807-8814.

8. Zhang X, Levy D, Kainz V, et al. Activation of central trigeminovascular neurons by cortical spreading depression. Ann Neurol 2011; 69: 855-865.

9. Russell MB and Olesen J. Increased familial risk and evidence of genetic factor in migraine. BMJ 1995; 311: 541-544.

10. Stewart WF, Staffa J, Lipton RB, et al. Familial risk of migraine: A population-based study. Ann Neurol 1997; 41: 166-172.

11. Ulrich V, Gervil M, Fenger K, et al. The prevalence and characteristics of migraine in twins from the general population. Headache 1999; 39: 173-180.

12. Gervil M, Ulrich V, Kyvik KO, et al. Migraine without aura: A population-based twin study. Ann Neurol 1999; 46: 606-611.

13. Mulder EJ, Van Baal C, Gaist D, et al. Genetic and environmental influences on migraine: A twin study across six countries. Twin Res 2003; 6: 422-431.

14. Ophoff RA, Terwindt GM, Vergouwe MN, et al. Familial hemiplegic migraine and episodic ataxia type- 2 are caused by mutations in the $\mathrm{Ca} 2+$ channel gene CACNL1A4. Cell 1996; 87: 543-552.

15. De Fusco M, Marconi R, Silvestri L, et al. Haploinsufficiency of ATP1A2 encoding the $\mathrm{Na}+/ \mathrm{K}+$ pump alpha2 subunit associated with familial hemiplegic migraine type 2. Nat Genet 2003; 33: 192-196.

16. Dichgans M, Freilinger T, Eckstein G, et al. Mutation in the neuronal voltage-gated sodium channel SCN1A in familial hemiplegic migraine. Lancet 2005; 366: 371-377.

17. Van den Maagdenberg AM, Haan J, Terwindt GM, et al. Migraine: Gene mutations and functional consequences. Curr Opin Neurol 2007; 20: 299-305.

18. Stam AH, Luijckx GJ, Poll-Thé BT, et al. Early seizures and cerebral edema after trivial head trauma associated with the CACNA1A S218L mutation. $J$ Neurol Neurosurg Psych 2009; 80: 1125-1129. 
19. Le Fort D, Safran AB, Picard F, et al. Elicited repetitive daily blindness: A new familial disorder related to migraine and epilepsy. Neurology 2004; 63: 348-350.

20. Vahedi K, Depienne C, Le Fort D, et al. Elicited repetitive daily blindness: A new phenotype associated with hemiplegic migraine and SCN1A mutations. Neurology 2009; 72: 1178-1183.

21. Zhuchenko O, Bailey J, Bonnen P, et al. Autosomal dominant cerebellar ataxia (SCA6) associated with small polyglutamine expansions in the alpha 1A-voltage-dependent calcium channel. Nat Genet 1997; 15: 62-69.

22. Gambardella A and Marini C. Clinical spectrum of SCN1A mutations. Epilepsia 2009; 50(Suppl 5): 20-23.

23. Thomsen LL, Ostergaard E, Olesen J, et al. Evidence for a separate type of migraine with aura: Sporadic hemiplegic migraine. Neurology 2003; 60: 595-601.

24. de Vries B, Freilinger T, Vanmolkot KR, et al. Systematic analysis of three FHM genes in 39 sporadic patients with hemiplegic migraine. Neurology 2007; 69: 2170-2176.

25. Terwindt $G$, Kors E, Haan J, et al. Mutation analysis of the CACNA1A calcium channel subunit gene in 27 patients with sporadic hemiplegic migraine. Arch Neurol 2002; 59: 1016-1018.

26. Thomsen LL, Oestergaard E, Bjornsson A, et al. Screen for CACNA1A and ATP1A2 mutations in sporadic hemiplegic migraine patients. Cephalalgia 2008; 28: 914-921.

27. Gallanti A, Cardin V, Tonelli A, et al. The genetic features of 24 patients affected by familial and sporadic hemiplegic migraine. Neurol Sci 2011; 32: S141-S142.

28. Riant F, Ducros A, Ploton C, et al. De novo mutations in ATP1A2 and CACNA1A are frequent in early-onset sporadic hemiplegic migraine. Neurology 2010; 75: 967-972.

29. De Sanctis S, Grieco GS, Breda L, et al. Prolonged sporadic hemiplegic migraine associated with a novel de novo missense ATP1A2 gene mutation. Headache 2011; 51: $447-450$

30. Russell MB and Ducros A. Sporadic and familial hemiplegic migraine: Pathophysiological mechanisms, clinical characteristics, diagnosis, and management. Lancet Neurol 2011; 10: 457-470.

31. Stam AH, Louter MA, Haan J, et al. A long-term followup study of 18 patients with sporadic hemiplegic migraine. Cephalalgia 2011; 31: 199-205.

32. Nyholt DR, LaForge KS, Kallela M, et al. A high-density association screen of 155 ion transport genes for involvement with common migraine. Hum Mol Genet 2008; 17: 3318-3331.

33. Cavalleri GL, Weale ME, Shianna KV, et al. Multicentre search for genetic susceptibility loci in sporadic epilepsy syndrome and seizure types: A case-control study. Lancet Neurol 2007; 6: 970-980.

34. Pietrobon D. Insights into migraine mechanisms and CaV2.1 calcium channel function from mouse models of familial hemiplegic migraine. $J$ Physiol 2010; 588: 1871-1878.

35. Cao YQ, Piedras-Rentería ES, Smith GB, et al. Presynaptic $\mathrm{Ca} 2+$ channels compete for channel type-preferring slots in altered neurotransmission arising from $\mathrm{Ca} 2+$ channelopathy. Neuron 2004; 43: 387-400.

36. Tao J, Liu P, Xiao Z, et al. Effects of familial hemiplegic migraine type 1 mutation T666M on voltage-gated calcium channel activities in trigeminal ganglion neurons. J Neurophysiol 2012; 107: 1666-1680.

37. Tavraz NN, Friedrich T, Dürr KL, et al. Diverse functional consequences of mutations in the $\mathrm{Na}+/ \mathrm{K}+-$ ATPase alpha2-subunit causing familial hemiplegic migraine type 2. J Biol Chem 2008; 283: 31097-31106.

38. Kahlig KM, Rhodes TH, Pusch M, et al. Divergent sodium channel defects in familial hemiplegic migraine. Proc Natl Acad Sci USA 2008; 105: 9799-9804.

39. Cestele S, Scalmani P, Rusconi R, et al. Self-limited hyperexcitability: Functional effect of a familial hemiplegic migraine mutation of the Nav1.1 (SCN1A) Na+ channel. J Neurosci 2008; 28: 7273-7283.

40. Castro MJ, Stam AH, Lemos C, et al. First mutation in the voltage-gated Nav1.1 subunit gene SCN1A with cooccurring familial hemiplegic migraine and epilepsy. Cephalalgia 2009; 29: 308-313.

41. Somjen GG. Mechanisms of spreading depression and hypoxic spreading depression-like depolarization. Physiol Rev 2001; 81: 1065-1096.

42. Van den Maagdenberg AM, Pietrobon D, Pizzorusso T, et al. A Cacnala knockin migraine mouse model with increased susceptibility to cortical spreading depression. Neuron 2004; 41: 701-710.

43. Van den Maagdenberg AM, Pizzorusso T, Kaja S, et al. High CSD susceptibility and migraine-associated symptoms in CaV2.1 S218L mice. Ann Neurol 2010; 67: 85-98.

44. Langford DJ, Bailey AL, Chanda ML, et al. Coding of facial expressions of pain in the laboratory mouse. Nat Methods 2010; 7: 447-449.

45. Eikermann-Haerter K, Dilekoz E, Kudo C, et al. Genetic and hormonal factors modulate spreading depression and transient hemiparesis in mouse models of familial hemiplegic migraine type $1 . J$ Clin Invest 2009; 119: 99-109.

46. Tottene A, Conti R, Fabbro A, et al. Enhanced excitatory transmission at cortical synapses as the basis for facilitated spreading depression in $\mathrm{Ca}(\mathrm{v}) 2.1$ knockin migraine mice. Neuron 2009; 61: 762-773.

47. Leo L, Gherardini L, Barone V, et al. Increased susceptibility to cortical spreading depression in the mouse model of familial hemiplegic migraine type 2. PLoS Genet 2011; e1002129.

48. James PF, Grupp IL, Grupp G, et al. Identification of a specific role for the $\mathrm{Na}, \mathrm{K}-\mathrm{ATPase}$ alpha 2 isoform as a regulator of calcium in the heart. Mol Cell 1999; 3: $555-563$.

49. Anttila V, Stefansson H, Kallela M, et al. Genome-wide association study of migraine implicates a common susceptibility variant on 8q22.1. Nat Genet 2010; 42: 869-873. **The first genome-wide association study (GWAS) in migraine, associating rs1835740 with migraine with aura. An eQTL analysis shows that the associated allele of the SNP is correlated with higher gene expression of MTDH. 
50. Dallas M, Boycott HE, Atkinson L, et al. Hypoxia suppresses glutamate transport in astrocytes. J Neurosci 2007; 27: 3946-3955.

51. Sarkar D, Park ES, Emdad L, et al. Molecular basis of nuclear factor-kappaB activation by astrocyte elevated gene-1. Cancer Res 2008; 68: 1478-1484.

52. Kang DC, Su ZZ, Sarkar D, et al. Cloning and characterization of HIV-1-inducible astrocyte elevated gene-1, AEG-1. Gene 2005; 353: 8-15.

53. Boycott HE, Wilkinson JA, Boyle JP, et al. Differential involvement of TNF alpha in hypoxic suppression of astrocyte glutamate transporters. Glia 2008; 56: 998-1004.

54. Andreou AP and Goadsby PJ. Therapeutic potential of novel glutamate receptor antagonists in migraine. Expert Opin Investig Drugs 2009; 18: 789-803.

55. Chasman DI, Schürks M, Anttila V, et al. Genome-wide association study reveals three susceptibility loci for common migraine in the general population. Nat Genet 2011; 43: 695-698. **The second GWAS in migraine, identifying variants near TRPM8, PRDM16 and LRP1 to be associated with migraine in a population-based study.

56. Peier AM, Moqrich A, Hergarden AC, et al. A TRP channel that senses cold stimuli and menthol. Cell 2002; 108: 705-715.

57. Proudfoot CJ, Garry EM, Cottrell DF, et al. Analgesia mediated by the TRPM8 cold receptor in chronic neuropathic pain. Curr Biol 2006; 16: 1591-1605.

58. Dray A. Neuropathic pain: Emerging treatments. $\mathrm{Br} J$ Anaesth 2008; 101: 48-58.

59. Cassé F, Bardou I, Danglot L, et al. Glutamate controls tPA recycling by astrocytes, which in turn influences glutamatergic signals. J Neurosci 2012; 32: 5186-5199.

60. Seale P, Bjork B, Yang W, et al. PRDM16 controls a brown fat/skeletal muscle switch. Nature 2008; 454: 961-967.

61. Kinameri E, Inoue $\mathrm{T}$, Aruga $\mathrm{J}$, et al. Prdm protooncogene transcription factor family expression and interaction with the Notch-Hes pathway in mouse neurogenesis. PLoS One 2008; 3: e3859.

62. Endo K, Karim MR, Taniguchi H, et al. Chromatin modification of Notch targets in olfactor receptor neuron diversification. Nat Neurosci 2011; 15: 224-233.

63. Freilinger T, Anttila V, de Vries B, et al. Genome-wide association analysis identifies susceptibility loci for migraine without aura. Nat Genet 2012; 44: 777-782.

**The third GWAS in migraine, associating four new variants in and near MEF2D, TGFBR2, PHACTR1 and $A S T N 2$ with migraine in a clinic-based cohort of migraine without aura patients and confirming TRPM8 and LRP1 as migraine susceptibility genes.

64. Flavell SW, Kim TK, Gray JM, et al. Genome-wide analysis of MEF2 transcriptional program reveals synaptic target genes and neuronal activity-dependent polyadenylation site selection. Neuron 2008; 60: 1022-1038.

65. Schytz HW, Birk S, Wienecke T, et al. PACAP38 induces migraine-like attacks in patients with migraine without aura. Brain 2009; 132: 16-25.

66. Law C, Bunyan D, Castle B, et al. Clinical features in a family with an $\mathrm{R} 460 \mathrm{H}$ mutation in transforming growth factor beta receptor 2 gene. J Med Genet 2006; 43: 908-916.

67. Jarray R, Allain B, Borriello L, et al. Depletion of the novel protein PHACTR-1 from human endothelial cells abolishes tube formation and induces cell death receptor apoptosis. Biochimie 2011; 93: 1668-1675.

68. Wang K, Li M and Hakonarson H. Analysing biological pathways in genome-wide association studies. Nat Rev Genet 2010; 11: 843-854.

69. Müller B and Grossniklaus U. Model organisms - A historical perspective. J Proteomics 2010; 73: 2054-2063.

70. Brennand KJ and Gage FH. Concise review: The promise of human induced pluripotent stem cell-based studies of schizophrenia. Stem Cells 2011; 29: 1915-1922.

71. De Vries B, Frants RR, Ferrari MD, et al. Molecular genetics of migraine. Hum Genet 2009; 126: 115-132.

72. Wessman M, Kallela M, Kaunisto MA, et al. Susceptibility locus for migraine with aura, on chromosome 4q24. Am J Hum Genet 2002; 70: 652-662.

73. Björnsson A, Gudmundsson G, Gudfinnsson E, et al. Localization of a gene for migraine without aura to chromosome 4q21. Am J Hum Genet 2003; 73: 986-993.

74. Nyholt DR, Morley KI, Ferreira MA, et al. Genomewide significant linkage to migrainous headache on chromosome 5q21. Am J Hum Genet 2005; 77: 500-512.

75. Anttila V, Kallela M, Oswell G, et al. Trait components provide tools to dissect the genetic susceptibility of migraine. Am J Hum Genet 2006; 79: 85-99. **A novel approach using trait component analysis (i.e. using individual migraine sub-traits) instead of 'end diagnoses' to determine disease status in a linkage analysis of Finnish families. Several chromosomal loci were identified.

76. Anttila V, Nyholt DR, Kallela M, et al. Consistently replicating locus linked to migraine on 10q22-q23. Am J Hum Genet 2008; 82: 1051-1063.

77. Nyholt DR, Gillespie NG, Heath AC, et al. Latent class and genetic analysis does not support migraine with aura and migraine without aura as separate entities. Genet Epidemiol 2004; 26: 231-244. **This study describes a latent class analysis of migraine (i.e. clustering approach using data on multiple migraine symptoms) and identifies three major types of migraine.

78. Tuulio-Henriksson A, Haukka J, Partonen T, et al. Heritability and number of quantitative trait loci of neurocognitive functions in families with schizophrenia. Am J Med Genet 2002; 114: 483-490.

79. Rommelse NN, Altink ME, Martin NC, et al. Neuropsychological measures probably facilitate heritability research of ADHD. Arch Clin Neuropsychol 2008; 23: 579-591.

80. Rubino E, Ferrero M, Rainero I, et al. Association of the C677T polymorphism in the MTHFR gene with migraine: A meta-analysis. Cephalalgia 2009; 29: 818-825.

81. Schurks M, Rist PM and Kurth T. MTHFR 677C $>$ T and ACE D/I polymorphisms in migraine: A systematic review and meta-analysis. Headache 2010; 50: 588-599.

82. Kaunisto MA, Kallela M, Hamalainen E, et al. Testing of variants of the MTHFR and ESR 1 genes in 1798 Finnish 
individuals fails to confirm the association with migraine with aura. Cephalalgia 2006; 26: 1462-1472.

83. Todt U, Freudenberg J, Goebel I, et al. MTHFR C677T polymorphism and migraine with aura. Ann Neurol 2006; 60: $621-622$.

84. Goyette P, Sumner JS, Milos R, et al. Human methylenetetrahydrofolate reductase: Isolation of cDNA, mapping and mutation identification. Nat Genet 1994; 7: 195-200.

85. Oterino A, Toriello M, Valle N, et al. The relationship between homocysteine and genes of folate-related enzymes in migraine patients. Headache 2010; 50: 99-168.

86. Suzuki M, Van Paesschen W, Stalmans I, et al. Defective membrane expression of the $\mathrm{Na}(+)-\mathrm{HCO}(3)(-)$ cotransporter NBCel is associated with familial migraine. Proc Natl Acad Sci USA 2010; 107: 15963-15968.

87. Igarashi $\mathrm{T}$, Sekine $\mathrm{T}$ and Watanabe $\mathrm{H}$. Molecular basis of proximal renal tubular acidosis. J Nephrol 2002; Suppl 5): S135-S141.

88. Lafrenière RG, Cader MZ, Poulin JF, et al. A dominantnegative mutation in the TRESK potassium channel is linked to familial migraine with aura. Nat Med 2010; 16: $1157-1160$.

89. Andres-Enguix I, Shang L, Stansfeld PJ, et al. Functional analysis of missense variants in the TRESK (KCNK18) K channel. Sci Rep 2012; 2: 237.

90. Bamshad MJ, Ng SB, Bigham AW, et al. Exome sequencing as a tool for Mendelian disease gene discovery. Nat Rev Genet 2011; 12: 745-755.

91. Lafrenière RG and Rouleau GA. Migraine: Role of the TRESK two-pore potassium channel. Int J Biochem Cell Biol 2011; 43: 1533-1536.

92. Tulleuda A, Cokic B, Callejo G, et al. TRESK channel contribution to nociceptive sensory neurons excitability: Modulation by nerve injury. Mol Pain 2011; 7: 30.

93. Stam AH, Luijckx GJ, Poll-Thé BT, et al. Early seizures and cerebral oedema after trivial head trauma associated with the CACNA1A S218L mutation. J Neurol Neurosurg Psychiatry 2009; 80: 1125-1129.

94. Kuhlenbäumer G, Hullmann J and Appenzeller S. Novel genomic techniques open new avenues in the analysis of monogenic disorders. Hum Mutat 2011; 32: 144-151.

**This describes the possibilities of novel techniques such as next-generation sequencing (NGS) for research purposes on monogenic disorders.
95. Ng SB, Buckingham KJ, Lee C, et al. Exome sequencing identifies the cause of a mendelian disorder. Nat Genet 2010; 42: 30-35. **This paper describes the first successful application of NGS in gene identification for a Mendelian disorder. In four patients with the recessive disorder Miller syndrome, previously unknown homozygous mutations were found in the $\mathrm{DHODH}$ gene. Sanger sequencing identified mutations in $\mathrm{DHODH}$ in three additional families.

96. Bourgeois M, Aicardi J and Goutieres F. Alternating hemiplegia of childhood. J Pediatr 1993; 122: 673-679.

97. Heinzen EL, Swoboda KJ, Hitomi Y, et al. De novo mutations in ATP1A3 cause alternating hemiplegia of childhood. Nat Genet 2012; 44: 1030-1034.**

98. Rosewich H, Thiele H, Ohlenbusch A, et al. Heterozygous de-novo mutations in ATP1A3 in patients with alternating hemiplegia of childhood: A whole-exome sequencing gene-identification study. Lancet Neurol 2012; 11: 764-773.**

** References 97 and 98 describe the successful application of NGS in the identification of de novo ATP1A3 mutations in approximately $\mathbf{7 0} \%$ of cases of the rare disorder $\mathrm{AHC}$.

99. Manolio TA, Collins FS, Cox NJ, et al. Finding the missing heritability of complex diseases. Nature 2009; 461: 747-753. **This is an outstanding review on the possible sources of the "missing heritability" (i.e. the approximately $80 \%$ of the heritability of common traits that are not detected in GWAS).

100. Thomsen LL and Olesen J. Nitric oxide in primary headaches. Curr Opin Neurol 2001; 14: 315-321.

101. Walsh T, McClellan JM, McCarthy SE, et al. Rare structural variants disrupt multiple genes in neurodevelopmental pathways in schizophrenia. Science 2008; 320: 539-543. **This article describes the increased occurrence of rare microdeletions and microduplications in schizophrenia patients that, in patients, disrupt genes involved in neurodevelopmental signaling. Structural variants may explain part of the missing heritability, as they are not detected in GWAS.

102. Klein RJ, Zeiss C, Chew EY, et al. Complement factor $\mathrm{H}$ polymorphism in age-related macular degeneration. Science 2005; 308: 385-389. 